\title{
OPEN SETS OF CONSERVATIVE MATRICES ${ }^{1}$
}

\section{DAVID BERG}

In this paper we present two principal results. In Theorem 1 we show that if $\Gamma^{l}$ denotes the open set of (conservative) matrices which map some subspace of $c$ of infinite deficiency isomorphically onto $c$ and if $\Lambda$ denotes the closed set of matrices which sum some bounded divergent sequence, then $\left[\Gamma^{l}\right]^{-}=\Lambda$. In Theorem 2 we produce a class of triangular matrices with the property that no triangular matrix in a neighborhood of one of these matrices has a range, as an operator on $m$, whose closure includes $c$.

We acknowledge valuable discussions with Dr. B. Johnson of Exeter University, formerly of Yale University.

We first introduce some notation, most of which is quite standard.

We denote by $m$ the Banach space of bounded sequences of complex numbers, where $\|x\|=\sup _{n}|x(n)|$. We denote by $c$ the subspace of $m$ consisting of convergent sequences, by $c_{0}$ the subspace of $c$ consisting of sequences with limit 0 , and by $E^{\infty}$ the (nonclosed) subspace of $c_{0}$ consisting of sequences with only a finite number of nonzero terms.

We denote by $\Gamma$ the Banach algebra of conservative matrices ( $A$ is called conservative if $x \in c \Rightarrow A x \in c$ ), and by $\Delta$ the Banach algebra of conservative matrices with zeros above the principal diagonal.

We denote by $C_{A}$ the vector space of sequences (including unbounded sequences) which are summed by $A$, that is, transformed into convergent sequences by $A$. If $C_{A} \cap m \neq c$ we say $A \in \Lambda$.

When no confusion seems likely to arise we do not differentiate, for $A \in \Gamma$, between $A$ as a transformation from $c$ to $c$ and $A$ as a transformation from $m$ to $m$. In this regard we note that if $A \in \Gamma$ is considered as an operator from $m$ to $m$, from $c$ to $c$, or from $c_{0}$ to $c$, the norm is the same; indeed $\|A\|=\sup _{i} \sum_{j}\left|a_{i j}\right|$. Furthermore, we recall that, for $A \in \Gamma$, if $A^{-1}$ exists for $A$ as an operator on $m$, then $A^{-1}$ exists for $A$ as an operator on $c$ and, hence, since $A^{-1}$ is a matrix, $A^{-1} \in \Gamma$ as was shown by $\mathrm{A}$. Wilansky and K. Zeller [3] and by M. R. Parameswaran [5] or as can be seen from our Lemma 1.

We first present the following characterization of $\Lambda$.

Lemma 1. A. $\in \Lambda$ if and only if for $\epsilon>0$ and integer $n$ there exists $x \in E^{\infty}$ such that

Presented to the Society, January 23, 1964; received by the editors March 16, 1964.

${ }^{1}$ This research was supported in part by N. S. F. Grant GP-1814. 
1. $x(1)=x(2)=\cdots=x(n)=0$,

2. $\|x\|=1$,

3. $\|A x\|<\epsilon$.

Proof. Let $A \in \Lambda$. Then there exists $x \in m$ such that, if $T_{n} x$ denotes $x$ with its first $n$ entries replaced by 0 ,
1. $\left|\lim _{m \rightarrow \infty}\left(A T_{n} x\right)(m)\right|<1 / N<\epsilon / 4$,
2. $\left\|A T_{n}(x)\right\|>1$,

for all $n$.

A sequence of the form

$$
\sum_{n=1}^{N}(1 / n) T_{m_{n}} x-\sum_{n=N+1}^{2 N}(1 / n) T_{m_{n}} x
$$

for large enough $m_{n}$, will satisfy 1,2 , and 3 .

The converse is clear by an easy gliding hump construction.

For details the reader is referred to [1]. This completes the proof of Lemma 1.

Lemma 1 yields the result of Wilansky and Zeller and Parameswaran referred to in the introduction when we observe that $A \in \boldsymbol{\Lambda}$ can have no left inverse as an operator from $m$ to $m$. Hence, if $A^{-1}$ exists, then $A^{-1}(c)=c$.

Lemma 1 also immediately yields the easy result that $\Lambda$ is closed.

Let $A \in \Gamma$. Suppose there exists some $c_{1}$ of infinite deficiency in $c$ such that if the domain of $A$ is cut down to $c_{1}, A$ is an isomorphism (topological) from $c_{1}$ onto $c$; i.e., if we consider $A: c_{1} \rightarrow c, A$ is an isomorphism onto $c$. We will denote the set of such $A$ by $\Gamma^{l}$.

We will show that the open set $\Gamma^{l}$ is dense in $\Lambda$.

LEMMA 2. $\Gamma^{l}$ is open.

Proof. $\Gamma^{l}$ is clearly open, since if $A \in \Gamma^{l}, A: c \rightarrow c$ has a right inverse $A^{r}$ whose range is $c_{1}$. Hence, for some neighborhood $A \subset \Gamma$, say $V, B \in V$ implies $B A^{r}(c)=c$. Hence, $B\left(c_{1}\right)=c$.

\section{LEMMA 3. $\Gamma^{l} \subset \Lambda$.}

Proof. Let $A \subset \Gamma^{l}$. Choose an infinite linearly independent set of vectors of the form $x_{i}-y_{i}$ where $x_{i} \in c \sim c_{1}$ and $y_{i} \in c_{1}$ such that $A\left(x_{i}-y_{i}\right)=0$. It can be easily seen (cf. $\$ 3.7$ of [4]) that, by a proper choice of scalar $\lambda_{i}$,

$$
A\left[\sum_{i=1}^{\infty} \lambda_{i}\left(x_{i}-y_{i}\right)\right]=0 \text { and } \sum_{i=1}^{\infty} \lambda_{i}\left(x_{i}-y_{i}\right) \in m \sim c .
$$

THEOREM 1. $\left[\Gamma^{l}\right]^{-}=\left[\left(\Gamma^{l}\right)^{0}\right]^{-}=\Lambda$. 
Proof. By Lemmas 2 and $3, \Gamma^{l}=\left(\Gamma^{l}\right)^{0}$ and $\left[\Gamma^{l}\right]-C \Lambda$. Hence we must show that $\Gamma^{l}$ is dense in $\Lambda$ (which is closed) and our theorem is established.

Let $A \in \Lambda$. By Lemma 1 we may choose a succession of columns of $A, a^{j(0)}, \cdots, a^{j(n)}, \cdots$ as follows: Choose $j(0)$ so that, for some $x_{0} \in E^{\infty}$,

1. $x_{0}[j(0)]=r_{0}$, where $\left|r_{0}\right|=1$,

2. $\left\|A\left(x_{0}\right)\right\|<\epsilon / 4$.

Choose $j(k), k=1,2, \cdots$, so that, for some $x_{k} \in E^{\infty}$,

1. $x_{k}[j(k)]=r_{k}$, where $\left|r_{k}\right|=1$,

2. $\left\|A x_{k}\right\|<(\epsilon / 8)\left(1 / 2^{k}\right)$,

3. $x_{k}(n)=0$ if $x_{k-1}(m) \neq 0$ for some $m \geqq n$.

Now define $B \in \Gamma$ as follows: $b^{j(k)}=a^{j(k)}+(\epsilon / 4) \delta_{k}-A\left(x_{k}\right) / r_{k}$, $k=0,1,2, \cdots$, where $\delta_{0}$ denotes the constant sequence all of whose entries are 1 and $\delta_{k}, k=1,2, \cdots$, denotes the sequence with 1 in the $k$ th place and 0 elsewhere. $b^{l}=a^{l}$ for all other $l$.

It is clear that $B \in \Gamma$ since $B=A+A^{\prime}+A^{\prime \prime}$, where $A^{\prime} \in \Gamma$ is a compact operator and $A^{\prime \prime}$ is a submethod of the identity.

$$
\|A-B\|<\frac{\epsilon}{4}+\frac{\epsilon}{4}+\frac{\epsilon}{4} \sum_{k=0}^{\infty} \frac{1}{2^{k}}=\epsilon \text {. }
$$

To see that $B \in \Gamma^{l}$, observe that $B\left(x_{k}\right)=(\epsilon / 4) x_{k}[j(k)] \delta_{k}$ $=(\epsilon / 4) r_{k} \delta_{k}, k=0,1,2, \cdots$. Hence, if $y \in c,\|y\|=1$,

$$
B\left\{\left(r_{0} \lim y\right) x_{0}+\sum_{j=1}^{\infty}(y(j)-\lim y) r_{j} x_{j}\right\}=\frac{\epsilon}{4} y .
$$

If we denote the above pre-image of $y$ by $B^{r} y$, we see that $\left\|B^{r} y\right\|$ $<3 \cdot 4 / \epsilon$. Clearly, $B^{r}(c)$ is isomorphic to $c_{0}$. It is also clear that $\{j(k)\}$ can be chosen so as to insure $B^{r}(c)$ being of infinite deficiency in $c$.

However, we note that $B^{r}$, as defined above, cannot be realized by a matrix.

This completes the proof of Theorem 1.

At this point we note that if we let $\Gamma^{l m}$ denote the set of $A \in \Gamma$ such that $A: m_{1} \rightarrow m$ is a (topological) isomorphism onto $m$ from some $m_{1}$ of infinite deficiency in $m$, the arguments of Lemmas 2, 3 and Theorem 1 all go through to show that $\Gamma^{l m}$ is an open dense set included in $\Lambda$. It is rather easy to see that $\Gamma^{l m} \supset \Gamma^{l}$. To see that the inclusion is proper, consider $A \in \Gamma$ defined by

$$
(A x)(n)=x(2 n)-x(2 n-1) .
$$

It is clear that slight variants of the foregoing arguments lead to other dense open sets in $\Lambda$; e.g., the set of $A$ which map $m$ onto a 
finite deficiency subspace of $m$, but have infinite dimensional kernel. However, we will not weary the reader with a catalogue of essentially similar results.

We now restrict our attention to $\Delta$.

In $\Delta$, Theorem 1 does not hold; indeed, the set of matrices which are not 1-1 on $c$ is nowhere dense. In $\Delta$, as contrasted with $\Gamma$, the maximal group consists of all matrices whose range is all of $c$.

We are able to present a small class of matrices in $\Lambda \cap \Delta$ which are not approximable in $\Delta$ by matrices whose range closure is $c$, hence which are not on the boundary of the maximal group in $\Delta$. We note that, by Theorem 1, we may approximate such matrices in $\Gamma$ by matrices whose range is $c$.

J. Copping on p. 193 of [2] presented an example of an element of $\Delta \cap \Lambda$ which is not on the boundary of the maximal group of $\Delta$.

Copping's example belongs to the class of Nörlund matrices described in the next lemma.

Lemma 4. Let complex numbers $k, l$ be given, where $|k|>1,|l|=1$. Let $A$ be the Nörlund matrix corresponding to $(x-k)(x-l)$. That is:

$$
\begin{aligned}
a_{i, i} & =1, \\
a_{i+1, i} & =-(k+l), \\
a_{i+2, i} & =k l, \\
a_{i, j} & =0 \text { for all other } i, j .
\end{aligned}
$$

Let $x_{0}$ be defined by

$$
x_{0}(1)=1, x_{0}(2)=-l, x_{0}(k)=0 \text { for all other } k .
$$

Let $\epsilon>0$ be given. Then there exists $\delta>0$ such that for $B \in \Delta$,

1. $\left\|\begin{array}{l}B-A \|<\delta, \\ x-x_{0}\end{array}\right\|<\delta$

jointly imply

$$
\left|\frac{y(n+1)}{y(n)}-k\right|<\epsilon,
$$

where $y=y(n)$ is defined by $B y=x$. Observe that for small enough $\epsilon, y \notin m$.

Proof. It suffices to consider the case $l=1$. Consider $y$ defined by $B y=x$ where $B \in \Delta$.

Fix $n$. If we express $y(n+1)$ as $y(n+1)=(1+\eta) k y(n)$ and if we express $y(n+2)$ as $y(n+2)=k(1+\rho) y(n+1)=k^{2}(1+\rho)(1+\eta) y(n)$, the following equation defines $\rho$. 


$$
\begin{array}{r}
\sum_{j=1}^{n-1} b_{n+2, j} y(j)+\left(k+c_{1}\right) y(n)-\left[(k+1)+c_{2}\right] k(1+\eta) y(n) \\
+k^{2}\left(c_{3}+1\right)(1+\rho)(1+\eta) y(n)=x(n+2),
\end{array}
$$

where $c_{1}=\left(b_{n+2, n}-a_{n+2, n}\right), c_{2}=\left(b_{n+2, n+1}-a_{n+2, n+1}\right), c_{3}=\left(b_{n+2, n+2}-a_{n+2, n+2}\right)$.

Solving for $\rho$ we get

$$
\begin{aligned}
\rho= & \frac{\eta}{k\left(c_{3}+1\right)(1+\eta)} \\
& +\frac{k y(n)\left[(1+\eta) c_{2}-(k+k \eta) c_{3}\right]-\left(\sum_{j=1}^{n-1} b_{n+2, j} y(j)\right)+x(n+2)-c_{1} y(n)}{k^{2}\left(c_{3}+1\right)(1+\eta) y(n)} \\
= & \frac{\eta}{k\left(c_{3}+1\right)(1+\eta)} \\
& +R, \text { say (provided all denominators are nonzero). }
\end{aligned}
$$

Choose $\delta_{1}>0, \bar{\eta}>0$ so small that

1. $\bar{\eta}<\min (\epsilon,|k|-1)$,

2. $\left|c_{3}\right|<\delta_{1},|\eta|<\bar{\eta}$ imply $\left|\eta / k(1+\eta)\left(c_{3}+1\right)\right|<2|\eta| /(1+|k|)$.

Now choose $\delta, 0<\delta<\delta_{1}$, so small that

1. $\|B-A\|<\delta,\left\|x-x_{0}\right\|<\delta$ implies $\left|y_{2} / y_{1}-k\right|<\bar{\eta},\left|y_{3} / y_{2}-k\right|<\bar{\eta}$,

2. (i) $\left|c_{i}\right|,|x(n+2)|, \sum_{j=1}^{n-1}\left|b_{n+2, j}\right|<\delta$,

(ii) $1 / 2<|y(1)|<\cdots<|y(n)|$,

(iii) $|\eta|<\bar{\eta}$,

jointly imply

$$
R<\frac{1}{2}\left(\frac{\bar{\eta}}{2}-\frac{\bar{\eta}}{1+|k|}\right) .
$$

Let $\|B-A\|<\delta,\left\|x-x_{0}\right\|<\delta$, where $\delta$ is chosen as above. (Note that this guarantees that 2(i) holds.)

Suppose $y(j+1)=\left(1+\eta_{j}\right) k y(j)$, where $\left|\eta_{j}\right|<|\bar{\eta}|, 1 \leqq j \leqq n$.

We now show that $y(n+2)=(1+\rho) k y(n+1)$ for some $\rho \in C$ such that $|\rho|<\bar{\eta}$.

If $\eta_{n}$ is such that $\bar{\eta} / 2<\eta_{n}<\bar{\eta}$, then $|\rho|<|2 \eta /(1+|k|)|+|R|<|\eta|$. If $\eta_{n}$ is such that $\eta_{n} \leqq \bar{\eta} / 2$, then $|\rho|<\bar{\eta} / 2+|R|<\bar{\eta} / 2+\bar{\eta} / 2=\bar{\eta}$. This completes the proof of Lemma 4 .

Theorem 2. Let $A=B G D$ where $B, G, D \in \Delta$ and

1. $B$ and $D$ are invertible,

2. $G$ is the Nörlund matrix corresponding to $(x-k)(x-l)$, where $|k|>1$.

Then there is a neighborhood, $V$, of $A$ in $\Delta$ with the following property: 
For each $H \in V$, there exists some $f \in l_{1}$ such that $H^{*} f=0$, i.e., $f H=0$, where $f$ is written as a row matrix. Equivalently, for $H \in V,[H(m)]-D c_{0}$.

Proof. We first observe that it suffices to prove the theorem for neighborhoods of $G$. For, if $V$ is a small enough neighborhood of $A$, $V=B V^{\prime} D$ where $V^{\prime}$ is a neighborhood of $G$. But if $f H=0$ for some $H \in V^{\prime}, f B^{-1} \in l_{1}$ and $\left(f B^{-1}\right) F=0$ for $F=B H D$.

We now consider three cases. Cases 1 and 2 are well known.

Case 1. $|l|<1$. This case is clear since $G$ is now an isomorphism of $c$ onto $f^{\perp}$ where $f=\left(1, k^{-1}, k^{-2}, \cdots\right) \in l_{1}=c_{0}^{*}$. Hence there exists $V \subset \Delta$, indeed, $V \subset \Gamma$, such that, for $H \in V$, there exists $g \in l_{1}$ such that $H^{*} g=0$.

Case 2. $|l|>1$. This case is, similarly, clear since $G$ is now an isomorphism of $c$ onto $f^{\perp} \cap h^{\perp}$ for appropriate $h \in l_{1}$.

Case 3. $|l|=1$. In this case $G(c)$ is not closed in $c$ so the above arguments do not apply. Indeed, $G(c)$ is dense not closed in $f^{\perp}$ where $f=\left(1, k^{-1}, k^{-2}, \cdots\right)$. But, by Lemma 4 , there exists a neighborhood of $G, V \subset \Delta$, such that for $H \in V[H(m)]-D x_{0}$ where $x_{0}$ is as defined in Lemma 4. Hence there exists $f \in l_{1}$ such that $H^{*} f=0$. This completes the proof of Theorem 2 .

In cases 1 and 2 of Theorem 2 the conclusion holds even if neighborhoods were taken in $\Gamma$. It is also clear that the arguments in these cases, which are quite standard, did not depend on the degree of the polynomial.

However, Theorem 1 tells us that in case 3 Theorem 2 is false if neighborhoods are taken in $\Gamma$. While it seems likely that case 3 is independent of the degree of the polynomial, we have not been able to prove the equivalent theorem for higher degree polynomials.

Theorems 1 and 2 suggest the following question which we cannot answer.

If $A \in \Lambda$ and $A$ is $1-1$ on $c$, is $A$ on the boundary of the maximal group of $\Gamma$ ?

\section{REFERENCES}

1. I. David Berg, A Banach algebra criterion for Tauberian theorems, Proc. Amer. Math. Soc. 15 (1964), 648-652.

2. J. Copping, Mercerian theorems and inverse transformations, Studia Math. 21 (1962), 177-194.

3. A. Wilansky and $\mathrm{K}$. Zeller, The inverse matrix in summability: reversible methods, J. London Math. Soc. 32 (1957), 397-408.

4. S. Mazur and W. Orlicz, On linear methods of summability, Studia Math. 14 (1954), 129-160.

5. M. R. Parameswaran, On the reciprocal of a $k$-matrix, J. Indian Math. Soc. (N.S.) 20 (1956), 329-331.

\section{YALE UNIVERSITY}

